\title{
Solution of the Moisture Phase Transition Problem in the Insulation and Soils near Pipelines
}

\author{
Boris Aksenov ${ }^{1, *}$ and Svetlana Kryaknina ${ }^{1}$ \\ ${ }^{1}$ Industrial University of Tyumen, 625001 Volodarskogo str. 38, Tyumen, Russia
}

\begin{abstract}
The analysis of various approaches to the heat conductivity modeling in soils with phase transition based on Stefan problem is provided. Herein the analytical solution for Stefan problem is received only for special cases. The approximate methods of mathematical modeling of heat exchange in soils and construction materials based on the Stefan problem without initial data are offered. The found analytical solution can be a basis for approximate solutions of a wide range of heat exchange problems at harmonious oscillation of temperature on one of the borders. The mathematical apparatus for estimating problems solution of freezing and melting in areas with plane-parallel and axial symmetry is considered. Solutions for applied problems of constructions building and operation in the permafrost conditions are provided.
\end{abstract}

\section{Introduction}

The prediction for freezing and melting boundary evolution is necessary to enable the successful constructions operation and working out measures to prevent the permafrost degradation. Stefan problem is a traditional mathematical model of heat conductivity in soil with phase transition. For one-dimensional semi-infinite domain the problem is defined as follows (soil thawing process is considered).

$$
\begin{gathered}
\frac{\partial t_{1}}{\partial \tau}=a_{1}^{2} \frac{\partial^{2} t_{1}}{\partial x^{2}}, \quad 0<x<\xi, \\
\frac{\partial t_{2}}{\partial \tau}=a_{2}^{2} \frac{\partial^{2} t_{2}}{\partial x^{2}}, \quad \xi<x<\infty, \\
t_{1}(0, \tau)=f(\tau), \quad t_{2}(x, 0)=\varphi(x), \quad t_{1}(\xi, \tau)=t_{2}(\xi, \tau)=t_{*}, \\
\left|t_{2}(x, \tau)\right|<M, \quad M=\text { Const },
\end{gathered}
$$

\footnotetext{
Corresponding author: tgasu.aksenov.boris@mail.ru
} 


$$
\left.\lambda_{1} \frac{\partial t_{1}}{\partial x}\right|_{x=\xi}-\left.\lambda_{2} \frac{\partial t_{2}}{\partial x}\right|_{x=\xi}=\kappa w \rho \frac{d \xi}{d \tau}
$$

where $\tau$ is the time; $x$ is spatial coordinate; $\xi$ is the interphase boundary coordinate; $t_{l}$ is the temperature in the thawed soil zone; $t_{2}$ is the temperature in the frozen soil zone; $t_{*}$ is the phase transition temperature; $\lambda_{1}, a_{1}{ }^{2}, \lambda_{2}, a_{2}{ }^{2}$ are thermal conductivity coefficient and thermal diffusivity coefficient in thawed and frozen zones, respectively; $\rho$ is the specific gravity of the soil skeleton; $k$ is the latent heat of water melting; $w$ is specific soil humidity.

The limitation condition (4) is necessary to enable the uniqueness of the problem solution (1) - (4).

The problem (1) - (5) is solved to determine the thawing boundary; it is also included in the mathematical models describing the various cryogenic phenomena such as frost heaving.

Analytical solution of this problem was obtained only for some special cases. Method of successive approximations in can be used to solve a nonlinear problem class of thermal conductivity, including the Stefan problem. There are several numerical solution schemes for the Stefan problem. In the scientific literature there are works constantly offering new approaches to solving this problem.

The variety of used methods indirectly indicates that this problem is not yet solved. The singularities of the type $\delta$-function when $x=\xi$ complicates numerical solution. These singularities approximation introduces a poorly controlled error. This means there is no single numerical method suitable for all coefficients and boundary conditions, even for onedimensional problems.

Approximate solutions based on the simplification of the problem (1) - (5) are used in engineering practice.

Assuming that $\varphi(x)=t_{*}$, we get the so-called one-phase Stefan problem. It means that the solution should be sought only in the thawed zone, since when $x>\xi$ at any value of $\tau$ temperature is known as $t_{2}=t_{*}$.

Convenient analytical solutions are obtained for a single-phase problem when $f(\tau)=t_{c}=$ Const and for a two-phase problem when $\varphi(x)=$ Const.

Further simplification is possible at a quasistationary approach. The boundary $x=\xi$ is believed to move slowly enough so at any instant of time $\tau$ temperature field in the domain $0<x<\xi$ can be considered stationary.

Using quasistationary solution of a one-dimensional single-phase problem when $f(\tau)=t_{c}$ we receive the well-known Stefan formula. If $f(\tau) \neq$ Const, then various modifications of the first and second L.S. Leybenzon methods are used. Those methods are also based on quasistationary approach.

From the heat balance standpoint quasistationary assumption is equivalent to the assumption of zero heat capacity in the "upper" zone $(0<x<\xi)$. The temperature in this zone varies but the thermal energy change due to the temperature change is not taken into account in the solution. Under certain conditions, this results in uncontrolled errors.

Without dwelling on the various attempts to consider the heat capacity of the "upper" zone which are widely represented in the literature, the authors should note that quasistationary approach is popular as it offers the simplest form of the boundary value problem solution in the domain $0<x<\xi$. This same problem solution for the equation (1) has the form of a Fourier series, and is very inconvenient at the calculations. 
However, if $f(\tau)$ is a periodic function (daily, annual temperature variation of the ground surface), then the closed form solution of heat conduction problem without initial data can be used in the upper zone. [1-13]

\section{Simulation using heat conduction problem without initial values}

Instead of problem (1) - (5) the problem (6) - (9) is solved:

$$
\begin{aligned}
& \frac{\partial t_{1}}{\partial \tau}=a_{1}^{2} \frac{\partial^{2} t_{1}}{\partial x^{2}}, \quad 0<x<\xi, \\
& t_{2}(x, \tau)=t_{*}, \quad \xi<x<\infty, \\
& t_{1}(0, \tau)=A+B \cdot \sin (\omega \tau+\varepsilon), \\
& t_{1}(\xi, \tau)=t_{2}(\xi, \tau)=t_{*},
\end{aligned}
$$

where $A, B, \omega, \varepsilon$ are constants, .

When solving the problem (6) - (9) position of phase border $x=\xi$ is considered fixed when finding expression for $t_{1}(x, \tau)$. The law of the border movement $\xi(\tau)$ is found from the Cauchy problem solution for the ordinary differential equation.

$$
\left.\lambda_{1} \frac{\partial t_{1}}{\partial x}\right|_{x=\xi}=-\kappa w \rho \frac{d \xi}{d \tau}, \quad \xi(0)=0
$$

Taking into account analytical solutions (6) - (9) to find $\xi(\tau)$ we receive a problem:

$$
\begin{aligned}
& \quad \frac{d \xi}{d \tau}=-\frac{\lambda}{\kappa w \rho}\left[\frac{\sqrt{2} B p}{\sin ^{2} p \xi+s h^{2} p \xi}\left(\operatorname{shp} \xi \cos p \xi \cos \left(\omega \tau+\frac{\pi}{4}\right)-\operatorname{chp} \xi \sin p \xi \sin \left(\omega \tau+\frac{\pi}{4}\right)\right)-\frac{A}{\xi}\right], \\
& \xi(0)=0 . \\
& \text { where } p=\sqrt{\omega /\left(2 a^{2}\right)} .
\end{aligned}
$$

Heat loss through the wall of a building is usually calculated on average daily temperatures. Approximate methods based on quasistationary approach take into account the daily heat loss dynamics.

The above method allows to consider the influence of both non-stationary and nonlinear heat transfer in the wall on the building heat loss. All construction materials contain moisture, so the correct thermal calculation for winter conditions should take into account that daily variations in ambient air temperature lead to variations of interphase boundaries $\xi$.

The Joule heat evolved or absorbed has a damping effect, resulting in a temperature variation phase displacement of the inner and outer building walls surfaces. This displacement is significant and should be taken into consideration when setting daily heating mode. 
Variations in an external layer $0<x<\xi$ are steady in nature so they can be described with a problem without initial data (6) - (9).

The swing value of $\xi$ is small enough, so the problem of the phase boundary moving is solved in the single-phase formulation.

In a layer $\xi<x<H$ (where $H$ is wall thickness) the influence of external air variations is considerably weakened, first of all by the phase transition boundary, so it is advisable to take the quasistationary approximation, in it is considered that the thermal flow at $x=\xi$ is equal to a thermal flow at $x=H$.

According to the calculations the daily air temperature variations, under the above assumptions do not change the total heat loss of the building. However, these variations significantly influence upon the phase displacement in temperature when $x=0$ and $x=H$. Thus, this phase displacement is equal to the temperature phase displacement on the outer and inner wall surfaces. The phase displacement $\xi(\tau)$ referred to $t(\tau)$, which is mainly determined by the specific heat and humidity of the material, can be considered as material properties to develop economical heat supply schedule.

A problem without initial data is applied to modeling the temperature field in the pipes insulation when laying external heat supply networks, because the heat loss during winter is determined both by the average daily air temperature, and by the daily temperature variations.

The temperature field calculation method in the insulation layer is similar to the processes modeling methods in the frozen soil and in a flat wall, but inhere the heat transfer occurs in the domain with axial symmetry. Therefore, we used the procedure of reduction of axisymmetric problems to the problems with plane-parallel symmetry.

For external insulation layer we have a problem without initial data in cylindrical coordinates:

$$
\begin{gathered}
\frac{\partial t}{\partial \tau}=\frac{a^{2}}{x} \frac{\partial}{\partial x}\left(x \frac{\partial t}{\partial x}\right), \quad \xi<x<R, \\
t(x, \tau)=t_{*}, \\
t(R, \tau)=A+B \cdot \sin (\omega \tau+\varepsilon)=f(\tau),
\end{gathered}
$$

where $t$ is insulation temperature, $R$ is preinsulated tube radius, A is average daily temperature, $B, \omega, \varepsilon$ are the constants indicating daily temperature variations.

The auxiliary problem (15) - (17) is taken to solve the problems (12) - (14):

$$
\begin{gathered}
\frac{\partial u}{\partial \tau}=a^{2} \frac{\partial^{2} u}{\partial y^{2}}, \quad 0<y<\xi_{1}, \\
u\left(\xi_{1}, \tau\right)=t_{*}, \\
u(0, \tau)=A+B \cdot \sin (\omega \tau+\varepsilon)=f(\tau) .
\end{gathered}
$$


where $\xi_{1}=R-\xi$.

when substituting

$$
x=R-y
$$

Coincidence of boundary conditions in problems (12) - (14) and (15) - (17) takes place. Following, the corresponding stationary problems solutions are considered. Connection between stationary problems solution in the field with plane-parallel symmetry and the problem solution in axisymmetric field is established.

As the phase boundary $x=\xi$ moves slowly, temperatures field in the area $\xi<x<R$ at each moment of time $\tau$ is considered stationary, we get the approximate linking formula between $u(x, \tau)$ and $t(x, \tau)$ :

$$
t(x, \tau) \approx t_{*}-\left(t_{*}-f(\tau)\right) \frac{\operatorname{Ln}\left(1+(\rho-1) \cdot \frac{t_{*}-u(x, \tau)}{t_{*}-f(\tau)}\right)}{\operatorname{Ln} \rho}, \quad \rho=\frac{R}{\xi} .
$$

Stefan's condition on phase transition border is set in single-phase statement. So the law of the border movement is set by the equation:

$$
\left.\lambda \frac{\partial t}{\partial x}\right|_{x=\xi}=-\kappa \gamma w \frac{d \xi}{d \tau},
$$

where $\lambda$ is heat conductivity coefficient, $\gamma$ is the specific mass of material, $w$ is relative humidity.

In view of (19), equation (20) takes the form

$$
\frac{d \xi}{d \tau}=\left.\frac{\lambda}{k \gamma w} \cdot \frac{1-\rho}{\operatorname{Ln} \rho} \cdot \frac{\partial u}{\partial x}\right|_{x=\xi} .
$$

In $(20)-(21)$ the interphase surface change at changes $\xi$ isn't considered because variations $\xi$ are small.

This procedure is suitable for the areas with big daily air temperature variations, such as the North of Western Siberia.

Thus, the analytical solution of heat conductivity problem without initial data can be the base for approximate solutions of a wide class of heat transfer problems when the temperature variations on one of the boundaries is stationary harmonic in nature.

\section{Estimates for problems solution of freezing and thawing in areas with plane-parallel and axial symmetry}

The problem of definition of phase transition front can be solved by creating the narrowing estimates system.

If convergence of recurrent procedure of estimating is proved, then we get the specific structure solution with properties similar to properties of converging alternate series. 
Namely, on each step it is possible to receive maximum value of absolute error with the error sign known which is important for applied problems.

I llustrates method of constructing a narrowing estimating system for the freezing and thawing problems in the area with plane-parallel symmetry.

Next the mathematical apparatus will be considered which allows to apply this method to heat exchange problems in the area with axial symmetry. The heat transfer problem with phase transition in moist fine disperse soil in an infinite one-dimensional axisymmetric domain is given by:

$$
\begin{gathered}
\frac{\partial t}{\partial \tau}=a^{2} \frac{\partial^{2} t}{\partial r^{2}}+\frac{a^{2}}{r} \frac{\partial t}{\partial r}-\frac{\chi}{c} \frac{\partial w(t)}{\partial \tau} \\
t\left(r_{0}, \tau\right)=F(\tau), \quad t(r, 0)=0, \quad \tau>0, \quad r>r_{0}, \quad|t(r, \tau)|<M, \quad M=\text { const }>0, \quad F(0)=0,
\end{gathered}
$$

where $a^{2}=\frac{\lambda}{c}, t, c, \lambda, \tau, w, \chi$ are temperature, heat capacity, thermal conductivity, time, spatial coordinate, the content of unfrozen moisture, the latent heat of the phase transition of water accordingly.

For simplicity, we assume that $F(\tau)$ is monotonic function with limited variation differentiable at $\tau>0$. The first two addends on the right side of (22) form the product of the coefficient on the one-dimensional Laplace operator in cylindrical coordinates, $\frac{\chi}{c} \cdot \frac{\partial w}{\partial \tau}$ is the source function. Here we have $\frac{\partial w(t)}{\partial \tau}=\frac{d w(t)}{d t} \cdot \frac{\partial t}{\partial \tau}$, where $\frac{d w}{d t}>0$ is a function established for the given type of soil ("curve of unfrozen water").

For the integral representation of (22) - (23) we need the Green's function, and it has a simple form for only a semi-infinite flat domain.

It means that equation (22) can be formally regarded as an equation for a semi-infinite flat domain $r>r_{0}$.

Then the integral representation of the problem (22) - (23) has the form:

$$
t(r, \tau)=t_{0}(r, \tau)-\frac{\chi}{c} \int_{r_{0}}^{\infty} \int_{0}^{\tau} G(r, \xi, \tau-y) \cdot \frac{\partial w}{\partial y} d \xi d y+a^{2} \int_{r_{0}}^{\infty} \int_{0}^{\tau} \frac{\partial t}{\partial \xi} G(r, \xi, \tau-y) \frac{1}{\xi} d \xi d y,
$$

where $G(r, \xi, \tau-y)$ is Green's function, $t_{0}(r, \tau)$ is the homogeneous heat equation solution with the conditions (23).

Applying the formula of integration by parts allows to eliminate the partial derivatives under the integral sign. Thus, we get:

$$
\begin{aligned}
& t(r, \tau)=V(r, \tau)+\frac{\chi}{c} \int_{r_{0}}^{\infty} \int_{0}^{\tau} \psi(r, \xi, \tau-y) w(t(\xi, y)) d \xi d y-a^{2} \int_{r_{0}}^{\infty} \int_{0}^{\tau} K(r, \xi, \tau-y) t(\xi, y) d \xi d y- \\
& -\frac{a^{2}}{r_{0}} \int_{0}^{\tau} G\left(r, r_{0}, \tau-y\right) t\left(r_{0}, y\right) d y,
\end{aligned}
$$

where $\psi(r, \xi, \tau-y)$ and $K(r, \xi, \tau-y)$ are function expressing the partial derivatives of the Green's function. 
The integrated equation (25) has the form suitable for receiving the narrowing system of estimates of the problem solution (22) - (23). Suppose there are estimates $t_{1}, t_{2}$, which lead to $t_{1} \leq t \leq t_{2}$. Having defined $\psi_{1}, \psi_{2}$ and $K_{1}, K_{2}$ similar to replacement in work and using estimates $t_{1}, t_{2}$ for $t(r, \tau)$ in equality (25), we receive the improved borders $t_{3}, t_{4}$ from the following derivations:

$$
\begin{aligned}
& t_{3}(r, \tau)=V(r, \tau)+\frac{\chi}{c} \int_{r_{0}}^{\infty} \int_{0}^{\tau}\left\{\psi_{1}(r, \xi, \tau-y) w\left(t_{1}(\xi, y)\right)-\psi_{2}(r, \xi, \tau-y) w\left(t_{2}(\xi, y)\right)\right\} d \xi d y- \\
& -a^{2} \int_{r_{0}}^{\infty} \int_{0}^{\tau}\left\{K_{1}(r, \xi, \tau-y) t_{1}(\xi, \tau)-K_{2}(r, \xi, \tau-y) t_{2}(\xi, y)\right\} d \xi d y- \\
& -\frac{a^{2}}{r_{0}} \int_{0}^{\tau} G\left(r, r_{0}, \tau-y\right) t\left(r_{0}, y\right) d y . \\
& t_{4}(r, \tau)=V(r, \tau)+\frac{\chi}{c} \int_{r_{0}}^{\infty} \int_{0}^{\tau}\left\{\psi_{1}(r, \xi, \tau-y) w\left(t_{2}(\xi, y)\right)-\psi_{2}(r, \xi, \tau-y) w\left(t_{1}(\xi, y)\right)\right\} d \xi d y- \\
& -a^{2} \int_{r_{0}}^{\infty} \int_{0}^{\tau}\left\{K_{1}(r, \xi, \tau-y) t_{2}(\xi, \tau)-K_{2}(r, \xi, \tau-y) t_{1}(\xi, y)\right\} d \xi d y- \\
& -\frac{a^{2}}{r_{0}} \int_{0}^{\tau} G\left(r, r_{0}, \tau-y\right) t\left(r_{0}, y\right) d y .
\end{aligned}
$$

It's obvious that $t_{1} \leq t_{3} \leq t \leq t_{4} \leq t_{2}$. Using $t_{3}, t_{4}$ under formulas similar to (26) - (27), we obtain the estimates $t_{5}, t_{6}$, and then the procedure is repeated recursively.

\section{Results}

Daily variation of external air temperature should be taken into account when calculating thermal fields in soil, construction materials and pipe line insulation. The control of daily temperature dynamics allows to establish phase displacement of temperature variations in and out of the premises resulting in correct daily mode of heating arrangement.

The algorithm of the freezing and melting problem solution can be used in engineering calculations, and also for testing various approximate methods. It should be noted that the similar solution can be constructed both for one-dimensional region with the central symmetry. Such a solution is especially valuable at high-quality research of freezing and melting processes in spherical shape regions.

\section{Conclusion}

At practical calculations of thermal fields in soil, construction materials and thermal*/ insulation of pipes it is expedient to consider daily fluctuation of temperature of external air. The accounting of daily dynamics of temperature allows to establish shift of phases of fluctuations of temperature in and out of the room that allows to organize the daily mode of heating correctly.

The scheme of the solution of a problem of frost penetration and thawing can be used by method of assessment directly in engineering calculations, and also for establishment of accuracy of various approximate methods. It should be noted that the similar solution can be constructed both for one-dimensional area with the central symmetry. Such a solution is 
especially valuable at high-quality research of processes of frost penetration and thawing in areas of spherical shape.

\section{References}

1. A. Ebrahimi-Moghadam, M. Farzaneh-Gord, M. Deymi-Dashtebayaz, Journal of Natural Gas Science and Engineering, 34, 185-196 (2016)

2. V.V. Remizov, A.F. Shapoval, B.V. Moiseev, B.G. Aksenov, Osobennosti stroitel'stva ob'ektov v neftegazodobyvajushhih rajonah Zapadnoj Sibiri (Nedra, Moscow, 1996).

3. E. Rossi di Schio, S. Lazzari, A. Abbati, Applied Thermal Engineering, 102, 227-233 (2014)

4. V.S. Sastri, Underground Pipeline Corrosion, 127-165 (2014)

5. J. Serrano-Arellano, J.M. Riesco-Ávila, J.M. Belman-Flores, K. Aguilar-Castro, E.V. Macías-Melo, International Journal of Heat and Mass Transfer, 102, 26-35 (2016)

6. H. Mahgerefteh, P. Zhang, S. Brown, International Journal of Greenhouse Gas Control, 46, 39-47 (2016)

7. S. R.Allahkaram, M. Isakhani-Zakaria, M. Derakhshani, M. Samadian, H. SharifiRasaey, A. Razmjoo, Journal of Natural Gas Science and Engineering, 26, 453-460 (2015)

8. M. A. Ersöz, A. Yildiz, International Journal of Refrigeration, 64, 51-56 (2016)

9. R F.V.V. de Sousa, R.O. da Mota, J.P. Quintela, M.M. Vieira, I.C.P. Margarit, O.R. Mattos, Electrochimica Acta, 52(27), 7780-7785 (2007)

10. B. G., S. Song, A. Ghalambor, T. R. Lin, Offshore Pipelines, 2, 113-124 (2014)

11. Y. Başoğul, A. Keçebaş, Energy, 36(10), 6156-6164 (2011)

12. Ju. S. Danielyan, D.K. Kalieva, P.Yu. Tret'jakov, Neftjanoe hozjajstvo Neftedobyvajushhaja promyshlennost', 40-42 (2009)

13. Ju.A. Sigunov, RIO Surgutskogo gosudarstvennogo universiteta (2009) 